\title{
Dissemination of clonal Salmonella enterica serovar Typhimurium isolates causing salmonellosis in Mauritius
}

Issack, M. I.; Migura, Lourdes Garcia; Ramsamy, Veemala D.; Svendsen, Christina Aaby; Pornruangwong, Srirat ; Pulsrikarn, Chaiwat ; Hendriksen, Rene S.

\author{
Published in: \\ Foodborne Pathogens and Disease
}

Link to article, DOI:

10.1089/fpd.2012.1426

Publication date:

2013

Document Version

Publisher's PDF, also known as Version of record

Link back to DTU Orbit

Citation (APA):

Issack, M. I., Migura, L. G., Ramsamy, V. D., Svendsen, C. A., Pornruangwong, S., Pulsrikarn, C., \& Hendriksen, R. S. (2013). Dissemination of clonal Salmonella enterica serovar Typhimurium isolates causing salmonellosis in Mauritius. Foodborne Pathogens and Disease, 10(7), 618-623. https://doi.org/10.1089/fpd.2012.1426

\section{General rights}

Copyright and moral rights for the publications made accessible in the public portal are retained by the authors and/or other copyright owners and it is a condition of accessing publications that users recognise and abide by the legal requirements associated with these rights.

- Users may download and print one copy of any publication from the public portal for the purpose of private study or research.

- You may not further distribute the material or use it for any profit-making activity or commercial gain

- You may freely distribute the URL identifying the publication in the public portal 


\title{
Dissemination of Clonal Salmonella enterica Serovar Typhimurium Isolates Causing Salmonellosis in Mauritius
}

\author{
Mohammad I. Issack, Lourdes Garcia-Migura, ${ }^{2}$ Veemala D. Ramsamy, ${ }^{1}$ Christina A. Svendsen, ${ }^{2}$ \\ Srirat Pornruangwong, ${ }^{3}$ Chaiwat Pulsrikarn, ${ }^{3}$ and Rene S. Hendriksen ${ }^{2}$
}

\begin{abstract}
Salmonella enterica serotype Typhimurium is one of the leading causes of salmonellosis in Mauritius, where it has also been associated with outbreaks of foodborne illness. However, little is known about its molecular epidemiology in the country. This study was therefore undertaken to investigate the clonality and source of Salmonella Typhimurium in Mauritius by studying human, food, and poultry isolates by pulsed-field gel electrophoresis (PFGE) and antibiotic minimum inhibitory concentration determination. Forty-nine isolates collected between 2008 and 2011 were analyzed, including 25 stool isolates from foodborne illness outbreaks and sporadic gastroenteritis cases, four blood isolates, one postmortem colon isolate, 14 food isolates, and five poultry isolates. All isolates were pansusceptible to the 16 antibiotics tested, except for two isolates that were resistant to sulfamethoxazole and trimethoprim. Overall characterization of the isolates by PFGE digested with XbaI and $B \ln \mathrm{I}$ resulted in eight different patterns. The largest of the clusters in the composite dataset consisted of 20 isolates, including two raw chicken isolates, four poultry isolates, and nine human stool isolates from two outbreaks. A second cluster consisted of 18 isolates, of which 12 originated from human blood and stool samples from both sporadic and outbreak cases. Six food isolates were also found in this cluster, including isolates from raw and grilled chicken, marlin mousse, and cooked pork. One poultry isolate had a closely related PFGE pattern. The results indicate that one clone of Salmonella Typhimurium found in poultry has been causing outbreaks of foodborne illness in Mauritius and another clone that has caused many cases of gastrointestinal illness and bacteremia in humans could also be linked to poultry. Thus, poultry appears to be a major reservoir for Salmonella Typhimurium in Mauritius. Initiating on-farm control strategies and measures against future dissemination may substantially reduce the number of cases of salmonellosis in the country.
\end{abstract}

\section{Introduction}

$\mathbf{N}$ ONTYPHOIDAL SALMONELLAE (NTS) are among the most common causes of reported bacterial foodborne illness worldwide (Todd, 1997; Hohmann 2001; Swaminathan et al., 2006). In Mauritius, an upper-middle-income island nation of 1.25 million inhabitants in the Southwest Indian Ocean, Salmonella enterica has been the bacterial pathogen most frequently isolated from stools of patients for the last 12 years (Moussa, 2007; Ministry of Health and Quality of Life, 2009). Furthermore, Salmonella serogroup O:4 containing Salmonella enterica serovar Typhimurium has been the most common serogroup isolated from stools specimens in the country from 1998 to 2007, with a yearly number of isolates ranging from 28
(34\%) in 1999 to 131 (64\%) in 2007(unpublished data). Moreover, the yearly number of patients with stools positive for Salmonella serogroup O:4 doubled from an average of 54.4 per year from 1998 to 2006 to 104.7 per year from 2008 to 2010 (unpublished data).

Salmonella Typhimurium has also been isolated in several specimens of raw chicken and ready-to-eat food in Mauritius and has been the causative organism of many outbreaks of foodborne illness. However, apart from isolates from an outbreak of Salmonella Typhimurium caused by consumption of contaminated marlin mousse, little is known about the molecular epidemiology of this serotype in the country (Issack et al., 2009). Two studies previously compared a small number of human and animal isolates of NTS from Mauritius by

\footnotetext{
${ }^{1}$ Central Health Laboratory, Victoria Hospital, Candos, Mauritius.

${ }^{2}$ WHO Collaborating Centre for Antimicrobial Resistance in Foodborne Pathogens and European Union Reference Laboratory for Antimicrobial Resistance, National Food Institute, Technical University of Denmark, Kgs. Lyngby, Denmark.

${ }^{3}$ WHO National Salmonella and Shigella Center, National Institute of Health, Department of Medical Sciences, Ministry of Public Health, Bangkok, Thailand.
} 
random amplified polymorphic DNA (RAPD) and repetitive extragenic palindromic polymerase chain reaction (rep-PCR), but very limited information was obtained as the first study did not include any nonhuman Salmonella Typhimurium isolate (Khoodoo et al., 2002) and the second study included only one nonhuman presumptive Salmonella Typhimurium isolate (Albufera et al., 2009).

The objective of the present study was to investigate the degree of clonality among a subset of Salmonella Typhimurium isolates causing gastrointestinal illness and bacteremia in hospitalized patients from Mauritius utilizing pulsed-field gel electrophoresis (PFGE) and antimicrobial susceptibility testing by minimum inhibitory concentration (MIC) determination. Additionally, this study was intended to find biological and epidemiological evidence of the transmission to the consumer of Salmonella Typhimurium through consumption of chicken and chicken-related ready-to-eat food.

\section{Materials and Methods}

\section{Isolation of Salmonella from different sources}

The Central Health Laboratory (CHL) processes clinical samples sent for bacteriologic investigations from all government healthcare institutions in Mauritius as well as from some private medical clinics. Additionally, CHL also processes all food samples collected by Ministry of Health inspectors for microbiological testing.

All stool specimens were cultured on xylose lysine deoxycholate (XLD) agar (Oxoid, Basingstoke, UK) and in selenite enrichment broth (Oxoid, Basingstoke, UK) followed by subculture on XLD agar. Blood cultures were processed manually by standard procedures (Freeman, 1989). Presence of Salmonella in raw and in ready-to-eat food specimens were detected by pre-enrichment of $25 \mathrm{~g}$ in peptone water followed by enrichment in selenite broth and subculture on XLD agar (PHLS, 1995).

Salmonella was identified by growth on Kligler's Iron agar, carbohydrate fermentation tests and agglutination with polyvalent $\mathrm{O}$ and polyvalent phase 1 and 2 antisera (Mast, Bootle, United Kingdom; Remel, Dartford, United Kingdom). Carbohydrate fermentation tests were performed by inoculation of the suspect organism in a test tube containing glucose, lactose, sucrose, mannitol, or dulcitol in peptone water with Andrade's indicator, and an inverted Durham tube (Pedler et al., 2004).

All blood culture and food isolates were confirmed by API 20E (Bio-Mérieux, Marcy l’Etoile, France). API 20E were performed on stool isolates only if biochemical or serological results were equivocal.

\section{Selection of isolates}

Forty-six isolates of Salmonella serogroup O:4 collected in 2009-2011 and suspected to be Salmonella Typhimurium were selected and sent to the National Food Institute, DTU-FOOD, Denmark for additional characterization. They included 28 human isolates originating from blood culture $(n=5)$, stool $(n=22)$, and postmortem intestinal swab $(n=1)$. Thirteen isolates were obtained from food samples. Five isolates originated from postmortem examination of poultry and were referred to the CHL by the Animal Health Laboratory in the veterinary division of the Ministry of Agro Industry. Ad- ditionally, three Salmonella Typhimurium isolates from 2008 that were previously investigated following an outbreak caused by consumption of marlin mousse (Issack et al., 2009) were included in the study for comparison purposes. All Salmonella serogroup O:4 isolated from food from 2009 to 2011 were available for the study, but human isolates were not systematically saved. Thus, human isolates were selected based on availability and to ensure representation of stool and blood isolates associated with both sporadic and outbreak cases.

\section{Serotyping}

All 49 isolates were serotyped at the National Institute of Health, Bangkok, Thailand using slide agglutination with hyperimmune sera (S \& A reagents lab, Ltd., Bangkok, Thailand) characterizing the $\mathrm{O}$ and $\mathrm{H}$ antigens. The serotypes were assigned according to the Kauffmann-White scheme (Grimont, 2007).

\section{Antimicrobial susceptibility testing}

Susceptibility to 16 different antimicrobial agents was performed at DTU-FOOD, on all Salmonella isolates as MIC determinations according to the previously described method (Hendriksen, 2008). For cefotaxime, a clinical breakpoint of $>2 \mathrm{mg} / \mathrm{L}$ was used (www.eucast.org).

\section{PFGE}

All 49 Salmonella Typhimurium isolates were analyzed for genetic relatedness at DTU-FOOD by PFGE using XbaI according to the CDC PulseNet protocol (Ribot, 2002). Subsequently, 47 of the isolates were further subtyped by restriction enzyme BlnI, named XmaJI by the producer (Fermantas, Helsingborg, Sweden), to increase the discriminatory power of the clusters. Evaluation of the composite dataset using both

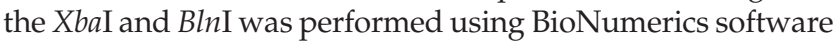
version 4.6 (Applied Maths, Sint-Martens-Latem, Belgium). The average from the experiments was used as settings for similarity and the unweighted-pair group method with arithmetic mean as dendrogram type.

\section{Results}

\section{Epidemiological data}

The epidemiological data are summarized in Figure 1. Three of the stool isolates (\#8286, \#8335, and \#8338) were obtained from children who developed gastroenteritis while staying in a hotel where several children became ill in December 2009. Chocolate mousse was thought to have been a likely vehicle of that outbreak, and although no leftover food was available for testing, Salmonella Typhimurium was isolated from chocolate peanut paste (\#1694) obtained in the hotel's kitchen. Stool isolates \#11S1068, \#11S1069, \#11S1076, and \#11S1103 originated from children who became ill at the same hotel in April 2011. Three stool isolates (\#8011, \#8303, and \#8309) originated from 10-11-year-old children who developed gastroenteritis 1 day after participating in a private school football tournament during which sandwiches with chicken ham and mayonnaise were served. One stool isolate (\#8235) was obtained 3 weeks later in a 1-year old sibling of a child who had participated in the tournament and was 


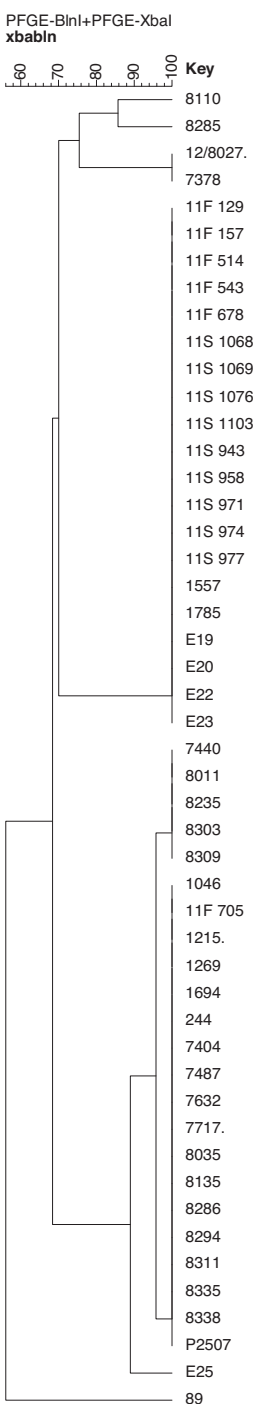

$\mathrm{m}^{*}$ : months

Origin
Human
Human
Human
Human
Pastry eclair
Pastry eclair
Pastry puits d'amour
Pastry eclair
Fresh raw chicken
Human
Human
Human
Human
Human
Human
Human
Human
Human
Raw chicken wings
Fresh raw chicken
Poultry
Poultry
Poultry
Poultry
Human
Human
Human
Human
Human
Raw marinated chicken
Cooked pork meat
Marlin mousse
Raw marinated chickenr
Chocolate peanut paste
Grilled chicken with mayonnaise
Human
Human
Human
Human
Human
Human
Human
Human
Human
Human
Human
Human
Poultry
Fresh raw chicken
Humation

Source
Stool
Stool
Stool
Blood
Food
Food
Food
Food
Food
Stool
Stool
Stool
Stool
Stool
Stool
Stool
Stool
Stool
Food
Food
Animal
Animal
Animal
Animal
Blood
Stool
Stool
Stool
Stool
Food
Food
Food
Food
Food
Food
Blood
Blood
Blood
Blood
Stool
Stool
Stool
Stool
Stool
Stool
Stool
Distal colon
Animal
Food

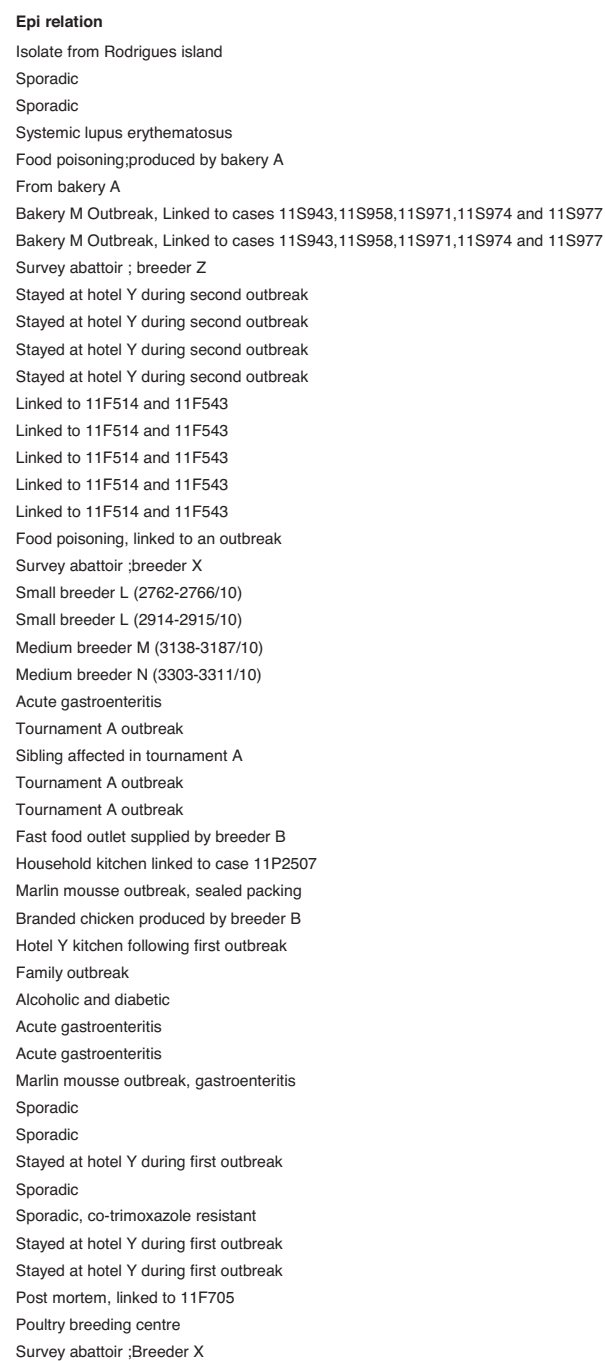

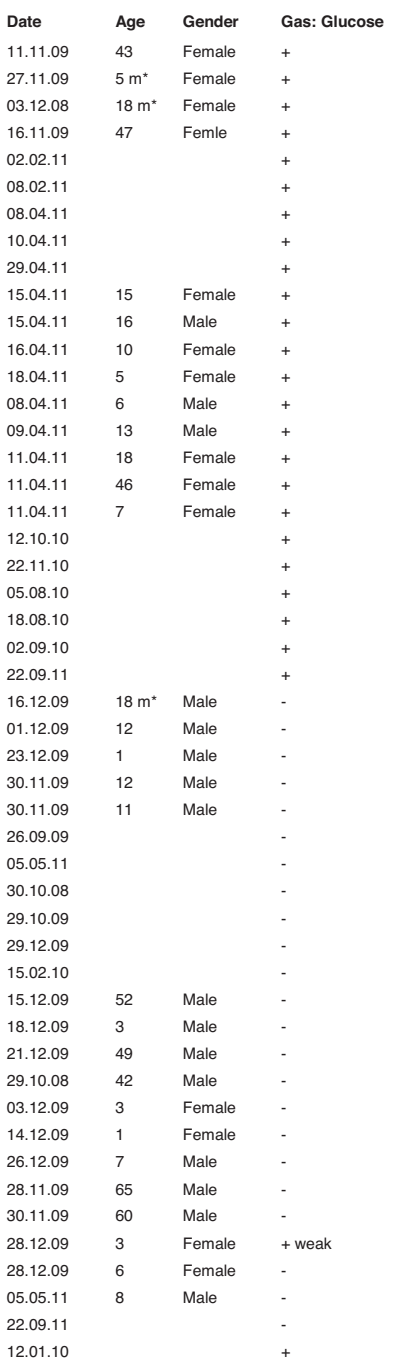

FIG. 1. Dendrogram showing the genotypic relatedness of the Salmonella enterica serovar Typhimurium isolates from Mauritius based on $\mathrm{XbaI}$ and $\mathrm{Bln} \mathrm{I}$ pulsed-field gel electrophoresis (PFGE) fingerprints.

reported to have had Salmonella isolated in his stools at a private laboratory. Stool isolates \#11S943, \#11S958, \#11S971, \#11S974, and \#11S977 originated from patients who became ill after eating cream cakes supplied by the same bakery, and food isolates \#11F514 and \#11S543 were obtained from cream cakes produced by that bakery. Food isolates \#11F129 and \#11F157 were obtained from cream cakes produced by another bakery. Isolate \#P2507 originated from a distal colon swab taken during postmortem examination of a child who died of gastroenteritis apparently associated with consumption of pork dumpling, and food isolate \#11F705 was obtained from a cooked pork specimen collected in the kitchen of the child's household. One of the stool isolates (\#8110) was a Salmonella Typhimurium referred to the CHL from Rodrigues, a tiny island in the Republic of Mauritius $500 \mathrm{~km}$ away from the main island.

One Salmonella Typhimurium isolate (\#1046) was obtained from a specimen of spiced raw chicken in October 2009 from a fast-food outlet and a second one (\#1269) originated, 2 weeks later, from a specimen of marinated raw chicken of a brand belonging to a major poultry breeder that also supplies the abovementioned fast-food outlet. No case of foodborne illness was associated with these two isolates. Food isolates \#89 and \#1785 originated from raw chicken sampled at an abattoir catering to small breeders and isolate \#11F678 was obtained from another such abattoir.

Poultry isolates \#E19 and \#E20 originated from a small breeder and isolates \#E22 and \#E23 were obtained from two medium breeders. Isolates \#E25 originated from poultry raised at the Poultry Breeding Centre of the Ministry of Agroindustry, which produces and sells poultry layers for small breeders.

\section{Biochemical fermentation and serotyping}

All isolates were identified as Salmonella Typhimurium. However, 23 of the 49 isolates did not produce gas after glucose fermentation, and one isolate (\#8335) was only a very weak producer of gas (Fig. 1). 


\section{Antimicrobial resistance}

All of the 49 Salmonella Typhimurium isolates included in the study were pansusceptible to ampicillin, apramycin, cefotaxime, ceftiofur, chloramphenicol, ciprofloxacin, florfenicol, gentamicin, nalidixic acid, neomycin, spectinomycin, sulfamethoxazole, tetracycline, and trimethoprim, except for two isolates (\#8311 and \#89) that were resistant to trimethoprim and sulfamethoxazole.

\section{PFGE typing}

The composite dataset of the PFGE typing with XbaI and $B \ln \mathrm{I}$ enzymes revealed eight unique PFGE patterns (Fig. 1). There were four distinct PFGE clusters, of which the largest consisted of 20 isolates that included all five stool and both food isolates associated with the large cream cake outbreak, two previous unrelated cream cake isolates linked with foodborne illness, and all four isolates linked with the second hotel outbreak. This cluster also contained two raw chicken isolates from different abattoirs, four poultry isolates from different small and medium breeders, and a raw chicken wing isolate associated with an episode of foodborne illness. The second largest cluster contained six food isolates, the three stool isolates from the first hotel outbreak, one blood isolate from the marlin mousse outbreak, one isolate from the distal colon swab, and seven blood and stool isolates from sporadic cases. The food isolates in this cluster originated from marlin mousse, hotel peanut butter paste, household cooked pork, grilled chicken with mayonnaise, spiced raw chicken from a fast-food outlet, and marinated raw chicken produced by the major breeder that supplies the fast-food outlet.

The third cluster contained five isolates, including those from the three children involved in the football tournament outbreak and from the sibling of a child affected by the outbreak. This cluster also included an 18-month-old child with no link to the tournament.

The last cluster of the composite dataset consisted of a blood isolate and a stool isolate with identical PFGE patterns. These two isolates were obtained almost 1 year apart from different patients.

Unique PFGE profiles were obtained for the isolate originating from the laboratory in Rodrigues Island, for a stool isolate from a sporadic case of gastroenteritis and for an abattoir raw chicken isolate.

The poultry isolate (\#E25) from the Poultry Breeding Centre had a unique PFGE profile that was related to those of the second and third clusters. Additionally, it also shared with isolates in these clusters the phenotypic characteristic of not producing gas from glucose.

\section{Discussion}

The PFGE results indicate that the Salmonella Typhimurium isolates from the food specimens are similar to those that have caused many outbreaks of foodborne illness, sporadic gastroenteritis, and bacteremia in Mauritius. Since 20 Salmonella Typhimurium isolates of the predominant PFGE cluster contained three and four specimens of raw chicken and dead poultry, respectively, poultry is its most probable reservoir. Similarly, poultry could potentially also be responsible for the Salmonella Typhimurium isolates included in the second most common PFGE cluster as it was found in two specimens of raw chicken originating from the same poultry breeder. Isolation of this clone from human blood, stool, and food sources at different points in time suggests that it might be well established in Mauritius. The presence of this clone from marlin mousse in 2008, chocolate peanut paste in 2009, and cooked pork in 2010 may have been caused by inadvertent crosscontamination in the kitchen with chicken. However, the possibility that this clone might also be present in other animal reservoirs or that contamination of food was caused by fecal carriers of this clone cannot be excluded. Contamination of peanut butter with Salmonella Typhimurium was associated with a well-documented outbreak in North America in 20082009 (Cavallaro et al., 2011), but any relation with the isolate from the chocolate peanut paste is unlikely as peanut paste available in Mauritius is imported from Europe, Asia, and South Africa rather than North America.

The five isolates from the third most common composite PFGE cluster were closely related to the second cluster and included three isolates that were directly related to an outbreak and a fourth originating from a secondary case in a household. The outbreak was notified late and no leftover food samples were available for testing; however, chicken, ham, or mayonnaise was thought to have been the likely vehicle of the Salmonella Typhimurium. This cluster may have evolved from the second cluster as a result of diversification during persistence of the organism in the reservoir (Soyer et al., 2010).

It is not surprising that a unique PFGE pattern was obtained from the isolate from Rodrigues Island, as inhabitants living there eat mostly vegetables, seafood, and occasionally meat from animals reared in the backyard, and unlike in Mauritius, chicken from large commercial farms is not part of the diet.

It is also noteworthy that one PFGE cluster was shared by two isolates that were not related in time or space. This would suggest that Salmonella Typhimurium with this PFGE fingerprint may be commonly encountered in Mauritius, but this could not be demonstrated clearly with the limited number of isolates typed.

After digestion with one restriction enzyme, a genetic variation does not always have an impact on the electrophoretic mobility of the restriction fragments during PFGE, and unrelated isolates may thus be incorrectly considered indistinguishable. Typing with a second enzyme was performed to improve the discriminatory ability of PFGE (Foley et al., 2007) and thus increase the likelihood that isolates with identical PFGE patterns are truly identical.

The increase in the number of Salmonella O:4 isolated in Mauritius in recent years suggests a rise in cases of salmonellosis caused by Salmonella Typhimurium. Chicken is the most commonly eaten meat in Mauritius and accounted for $70 \%$ of all meat consumption in 2009 (Central Statistics Office, 2009). However, changes in the diet cannot explain the increased number of human isolates, as chicken consumption only increased by $23 \%$ from 36,085 tons in 2006 to 44,420 tons in 2009 (Dr. Neerunjun, personal communication).

The PFGE results also showed that Salmonella Typhimurium isolates from blood were indistinguishable from many stool isolates. Unlike in sub-Saharan Africa where a distinct genotype, Salmonella Typhimurium ST 313, was found to cause invasive infections but was not thought to be a significant cause of diarrhea (Kingsley, 2009), in Mauritius, 
gastroenteritis and bacteremia are caused by the same clones of the organism.

None of the Salmonella Typhimurium isolates studied was multidrug resistant. In contrast to the situation in many countries (Glynn et al., 1998; Vandenberg et al., 2010), Salmonella Typhimurium in Mauritius remain remarkably susceptible to most antibiotics, with resistance rates in Salmonella serogroup O:4 isolated from 1998 to 2009 of around 5\% to ampicillin and co-trimoxazole, and $<1 \%$ to quinolones (unpublished data). It is difficult to obtain information from poultry producers on their use of antimicrobials, but the resistance data available suggest that antibiotics are not used widely. The main local producer of chicken feed insists that it follows European Union guidelines on the use of antimicrobials in animal feeds. Moreover, in Mauritius, import of all antibiotics, including those intended for use in veterinary practice, needs the authorization of the Director of Pharmaceutical Services of the Ministry of Health before they are cleared by customs services. NTS usually produce gas from glucose, but it was noted at the CHL that several isolates of Salmonella serogroup O:4 did not produce gas from glucose. In 2009, this phenotypic characteristic was found in all three food isolates, seven of the 10 blood isolates, and 41 (44\%) of 93 stools isolates (unpublished data). In the present study, 17 of 23 isolates that did not produce gas from glucose had the same PFGE pattern and the six other isolates had closely related PFGE patterns. Such biochemical characteristics can be useful in identifying suspect novel strains and for epidemiological investigations over short periods of time (Acha et al., 2001). However, like most phenotypic characteristics, they are inherently limited by the ability of microorganisms to alter the expression of the underlying genes, and such changes may occur unpredictably or in response to environmental stimuli (Arbeit, 1995). Indeed, in our study, one isolate with the same PFGE pattern was a weak producer of gas from glucose even though it was epidemiologically related to non-gas-producing isolates.

Our study on the epidemiology of Salmonella Typhimurium had certain limitations. We did not have any isolate from live poultry. Testing of specimens from live poultry is performed in the Animal Health Laboratory of the Ministry of Agro Industry but no Salmonella spp. were isolated in 2008 and 2009 (Dr. Neerunjun, personal communication). However, only a limited number of cloacal swabs collected from poultry were cultured, and the method may not have been sensitive enough. Another limitation was the nonavailability of all human isolates during the study period. Isolates associated with outbreaks were more likely to have been saved, although a few isolates associated with sporadic cases from certain periods were also available.

This study indicates that poultry is the probable reservoir for Salmonella Typhimurium isolated among humans in Mauritius. Control of Salmonella Typhimurium in broilers from affected poultry breeders could lead to a marked reduction in the number of cases of salmonellosis in humans. Control strategies should include the use of Salmonella-free parent flocks and Salmonella-free chicken feeds. Poultry houses should be cleaned and disinfected regularly, with empty resting periods between flocks, and measures should be taken to prevent domestic and wild animals, including wild birds, from gaining access to the premises. A more extreme and costly but effective strategy involves in- tensive flock-level testing with destruction of infected flocks (http://www.fao.org/DOCREP/MEETING/004/AB456E .HTM). However, apparently expensive measures could eventually be cost-effective, as reduction of Salmonella in food animals would most likely prevent many human cases of salmonellosis.

\section{Acknowledgments}

This work was supported by the World Health Organization Global Foodborne Infections Network (WHO GFN) (www.who.int/gfn). We would also like to thank Mrs. P. Lan Keng Lun, Mr. R.K. Lutchun, Miss N. Kanaksabee, and all the technical staff of the bacteriology section of the CHL for their outstanding technical assistance.

\section{Disclosure Statement}

No competing financial interests exist.

\section{References}

Acha PN, Szyfres B (eds.). Salmonellosis. In: Zoonoses and Communicable Diseases Common to Man and Animals: Bacterioses and Mycoses. $3^{\text {rd }}$ ed. Washington, DC: PAHO, 2001, pp. 233-246.

Albufera U, Bhugaloo-Vial P, Issack MI, Jaufeerally-Fakim Y. Molecular characterization of Salmonella isolates by REP-PCR and RAPD analysis. Infect Genet Evol 2009;9:322-327.

Arbeit RD. Laboratory procedures for the epidemiologic analysis of microorganisms. In: Manual of Clinical Microbiology, $6^{\text {th }}$ ed. Murray PR, Baron EJ, Pfaller MA, Tenover FC, Yolken RH (eds.). Washington, DC: ASM Press, 1995, pp. 190-208.

Cavallaro E, Date K, Medus C, Meyer S, Miller B, Kim C, Nowicki S, Cosgrove S, Sweat D, Phan Q, Flint J, Daly ER, Adams J, Hyytia-Trees E, Gerner-Smidt P, Hoekstra RM, Schwensohn C, Langer A, Sodha SV, Rogers MC, Angulo FJ, Tauxe RV, Williams IT, Behravesh CB; Salmonella Typhimurium Outbreak Investigation Team. Salmonella typhimurium infections associated with peanut products. $\mathrm{N} \mathrm{Engl} \mathrm{J} \mathrm{Med}$ 2011;365:601-610.

[CSO] Central Statistics Office. Ministry of Finance \& Economic Development. Digest of agricultural statistics. 2009. Available at: http://www.gov.mu/portal/goc/cso/report/natacc/agri09/ agri09.pdf, accessed November 5, 2012.

Foley SL, Zhao S, Walker RD. Comparison of molecular typing methods for the differentiation of Salmonella foodborne pathogens. Foodborne Pathog Dis 2007;4:253-276.

Freeman R. Bacteriology of normally sterile body fluids. In: Medical Bacteriology, $1^{\text {st }}$ ed. Hawkey P, Lewis D (eds.). New York: Oxford University Press, 1989, pp. 21-42.

Glynn MK, Bopp C, Dewitt W, Dabney P, Mokhtar M, Angulo FJ. Emergence of multidrug-resistant Salmonella enterica serotype typhimurium DT104 infections in the United States. N Engl J Med 1998;338:1333-1338.

Grimont PAD, Weill FX. Antigenic Formulae of the Salmonella Serovars, 2007, $9^{\text {th }}$ ed. Paris: Institut Pasteur, WHO Collaborating Center for Reference and Research on Salmonella, 2007.

Hendriksen RS, Bangtrakulnonth A, Pulsrikarn C, Pornreongwong S, Hasman H, Song AW, Aarestrup FM. Antimicrobial resistance and molecular epidemiology of Salmonella Rissen from animals, food products and patients in Thailand and Denmark. Foodborne Pathog Dis 2008;5:605-619.

Hohmann EL. Nontyphoidal salmonellosis. Clin Infect Dis 2001;32:263-269. 
Issack MI, Hendriksen RS, Lun PL, Lutchun RK, Aarestrup FM. Salmonella enterica serovar Typhimurium in Mauritius linked to consumption of marlin mousse. Foodborne Pathog Dis 2009;6:739-741.

Khoodoo MH, Issack MI, Jaufeerally-Fakim Y. Serotyping and RAPD profiles of Salmonella enterica isolates from Mauritius. Lett Appl Microbiol 2002;35:146-152.

Kingsley RA, Msefula CL, Thomson NR, Kariuki S, Holt KE, Gordon MA, Harris D, Clarke L, Whitehead S, Sangal V, Marsh K, Achtman M, Molyneux ME, Cormican M, Parkhill J, MacLennan CA, Heyderman RS, Dougan G. Epidemic multiple drug resistant Salmonella Typhimurium causing invasive disease in sub-Saharan Africa have a distinct genotype. Genome Res 2009;19:2279-2287.

Ministry of Health \& Quality of Life. Island of Mauritius Health Statistics Annual 2009. Pathological tests by (positivity rates) 2006-2008. Available at: http://www.gov.mu/portal/goc/ moh/file/statsm08/oth08m/p45.pdf, accessed November 5, 2012.

Moussa MF. Etude comparative des germes intestinaux isolés au laoratoire de référence de l'île Maurice entre 1998-99 et 200405. Diplôme Universitaire de santé publique à l'île Maurice. Université Victor Ségalen, Bordeaux 2 et Institut de Santé de Maurice, 2007. (In French.)

Pedler S, Graham G. Bacteriology of intestinal disease. In: Medical Bacteriolog, $2^{\text {nd }}$ ed. Hawkey P, Lewis D (eds.). New York: Oxford University Press, 2004, pp. 177-213.

[PHLS] Public Health Laboratory Service. Isolation and Enrichment of Micro-organisms. In: Practical Food Microbiology, $2^{\text {nd }}$ ed. Roberts D, Hooper W, Greenwood M (eds.). London: Public Health Laboratory Service, 1995, pp. 121-169.

Ribot EM, Wierzba RK, Angulo FJ, Barrett TJ. Salmonella enterica serotype Typhimurium DT104 isolated from humans, United States, 1985, 1990, and 1995. Emerg Infect Dis 2002;8:387-391. Soyer Y, Alcaine SD, Schoonmaker-Bopp DJ, Root TP, Warnick LD, McDonough PL, Dumas NB, Gröhn YT, Wiedmann M. Pulsed-field gel electrophoresis diversity of human and bovine clinical Salmonella isolates. Foodborne Pathog Dis 2010; 7:707-717.

Swaminathan B, Gerner-Smidt P, Barrett T. Focus on Salmonella. Foodborne Pathog Dis 2006;3:154-156.

Todd EC. Epidemiology of foodborne diseases: A worldwide review. World Health Stat Q 1997;50:30-50.

Vandenberg O, Nyarukweba DZ, Ndeba PM, Hendriksen RS, Barzilay EJ, Schirvel C, Bisimwa BB, Collard JM, Aidara Kane A, Aarestrup FM. Microbiologic and clinical features of Salmonella species isolated from bacteremic children in eastern Democratic Republic of Congo. Pediatr Infect Dis J 2010;29: 504-510.

Address correspondence to: Mohammad I. Issack, FRCPath Central Health Laboratory Victoria Hospital Candos, Mauritius

E-mail: moissack@yahoo.com 